\title{
An orthotropic interface damage model for simulating drying processes in soils
}

\author{
Osvaldo Manzoli ${ }^{1} \cdot$ Marcelo Sánchez $^{2} \cdot$ Michael Maedo $^{2} \cdot$ Jumanah Hajjat $^{2} \cdot$ Leonardo J. N. Guimarães $^{3}$
}

Received: 3 May 2017/Accepted: 15 November 2017/Published online: 8 December 2017

(C) Springer-Verlag GmbH Germany, part of Springer Nature 2017

\begin{abstract}
The study of drying process in soils has received an increased attention in the last few years. This is very complex phenomenon that generally leads to the formation and propagation of desiccation cracks in the soil mass. In recent engineering applications, high aspect ratio elements have proved to be well suited to tackle this type of problem using finite elements. However, the modeling of interfaces between materials with orthotropic properties that generally exist in this type of problem using standard (isotropic) constitutive model is very complex and challenging in terms of the mesh generation, leading to very fine meshes that are intensive CPU demanding. A novel orthotropic interface mechanical model based on damage mechanics and capable of dealing with interfaces between materials in which the strength depends on the direction of analysis is proposed in this paper. The complete mathematical formulation is presented together with the algorithm suggested for its numerical implementation. Some simple yet challenging synthetic benchmarks are analyzed to explore the model capabilities. Laboratory tests using different textures at the contact surface between materials were conducted to evaluate the strengths of the interface in different directions. These experiments were then used to validate the proposed model. Finally, the approach is applied to simulate an actual desiccation test involving an
\end{abstract}

Marcelo Sánchez

msanchez@civil.tamu.edu

1 Department of Civil Engineering, São Paulo State University (UNESP), São Paulo, Bauru, Brazil

2 Zachry Department of Civil Engineering, Texas A\&M University, College Station, TX 77845, USA

3 Department of Civil Engineering, Federal University of Pernambuco, Recife, Brazil orthotropic contact surface. In all the application cases the performance of the model was very satisfactory.

Keywords Drying cracks - Mesh fragmentation · Orthotropic interphase elements - Orthotropic shear strength $\cdot$ Shrinkage 3D FE modeling

\section{Introduction}

The use of interface elements in the context of finite element (FE) simulations is based on the idea of introducing special elements in between the standard (bulk) ones of a FE mesh [10, 21-23, 27, 32, 33, 40, 43]. For example, zero-thickness interface elements have been used to explicitly include the presence of discontinuities when modeling fractured rock masses [12]. This type of interface element has also been used in the meso-scale-level modeling of cracks and fractures in concrete $[6,7,9,19,20]$. Approaches based on similar concepts, as, e.g., cohesive zone models, have also been used in geo-engineering applications [26].

The formation and propagation of discontinuities in solids and porous media is a very challenging problem that is driving the development of both experimental $[16,18,28,34,38,39]$ and advanced numerical techniques $[2,3,14,31,42]$. In this context, the mesh fragmentation technique (MFT) in conjunction with high aspect ratio (HAR) elements has been used with success to model the problem of formation and grow of drying cracks in soils [40], as well as the problem of fracture generation and propagation in concrete structures [22, 23, 37]. Some advantages of this technique are discussed in Sect. 2.

The proper modeling of the contact between materials generally plays a critical role for a successful simulation of 
several problems, as for example, the shrinkage behavior of soils subjected to drying. Drying experiments show that the contact between the soil and the underlying (rigid) surface controls the formation of drying cracks, impacting in the spacing between them, their orientation and other characteristics of the crack network. For example, Peron et al. [34] reported that a clayey soil upon drying and under free shrinkage conditions did not crack. However, the same soil under identical environmental conditions, but with displacement restrictions at the soil-plate contact (rigid) surface did develop desiccation cracks. To reproduce free shrinkage conditions the soil sample was prepared in a rectangular Teflon plate, whereas metallic rectangular notches were included at the contact between the soil and the plate to impose the restriction in the longitudinal direction. The numerical analysis of this experiment presented in Sánchez et al. [40] also shows that the adhesion between soil and the (no-deformable) surface controls the spacing between drying cracks. Furthermore, to properly simulate the shrinkage-restricted test discussed above it was necessary to discretize every single notch present at the soil-plate contact surface.

An orthotropic interface mechanical model capable of distinguishing between strengths in different directions would highly facilitate the modeling of this type of problem. For example, it will minimize the time-consuming process of developing the meshes incorporating all the details of the contact surfaces that generally impact on directional adhesions and strengths. Furthermore, it will reduce the number of degree of freedoms of the problem (and therefore the associated CPU time), because the orthotropic characteristics of the contact surface(s) will be incorporated in the orthotropic mechanical model, and therefore, the fine mesh generally necessary to explicitly represent textured contact surfaces will not be necessary when the directional properties are implicitly contemplated into the interface constitutive model. The benefits of an orthotropic constitutive model do not limit to the simulation of the laboratory tests discussed above, because several engineering problems are affected by the presence of orthotropic interfaces. For example, some authors suggested the term 'unsaturated soil interface' [13] to identify the contact surface between the more superficial and degraded soil (i.e., the one subjected directly to climate variations) and the underlying ground. A proper modeling of this type of interface would contribute to realistic predictions of relevant engineering problems involving this condition, such as landslides, foundations, and slope stability. The application of the orthotropic interface model does not limit to soil mechanics only, because the presence of orthotropic surfaces has been reported in other engineering problems. For example, the study of orthotropic properties is very common in rock mechanics problems dealing with joints and discontinuities. The fracture roughness coefficient is based on an evaluation of the surface geometric properties, which generally varies with the direction of the analysis. In this context, Misra [25] proposed a micromechanical model for anisotropic discontinuities, and Li et al. [17] conducted an experimental research to study the anisotropic shear behavior of fractured rocks. Orthotropic models could also be useful for problems involving the analysis of indentation and microindentation in concrete [1].

A discrete anisotropic constitutive model was proposed by Ferrara and Pandolfi [10] and Pandolfi and Weinberg [33] to simulate the formation of cracks at interelemental surfaces of FE models dealing with anisotropic materials. The model was developed in the context of the zerothickness finite element theory. Although this discrete model shares some characteristics with the orthotropic continuum damage model presented here, that one [i.e., 10, 31] was designed to describe mixed-mode crack formation (with normal opening displacements), being not totally applicable to model contact and friction phenomena. Aspect that is required for describing the presence of interfaces between materials with orthotropic properties, as it occurs in the cases studied in this work.

This paper focuses on the formulation and application of a damage orthotropic interface constitutive model developed in the framework of the MFT for dealing with problems involving contact surfaces with orthotropic strength properties, and it is organized as follows. A brief review of the mesh fragmentation method is introduced first. Then, the mathematical framework of the proposed model is presented in detail together with the numerical algorithm suggested for its implementation in a FE code. Afterward, the model is checked by means of a number of synthetic benchmarks aimed at exploring the performance of the proposed approach under typical loading conditions. The validation of the orthotropic model against direct shear tests conducted to study the strength properties between soils and plates with different textures is discussed afterward. The final numerical analysis is related to the study of the drying process of soil subjected to orthotropic shrinkage restrictions. The paper closes with the main conclusion of this work.

\section{Mesh fragmentation technique}

A MFT based on the inclusion of solid HAR interface elements was recently proposed to model the formation and propagation of drying cracks in soils [40]. The high aspect ratio elements [22, 23] are incorporated in between the standard finite elements of a mesh. The main stages associated with the adaptation of a traditional FE mesh into a 
fragmented one are presented in Fig. 1. The regular elements of a standard FE mesh (Fig. 1a) are first separated by introducing gaps between them (Fig. 1b). These gaps are relatively small (typically around $0.01 \mathrm{~mm}$ ). As demonstrated in Manzoli et al. [22], the size of the gaps does not affect the numerical results. In Fig. 1b the spaces between elements are exaggerated to illustrate better the method. To complete the transformation of the standard FE mesh, HAR elements are placed to fill the gaps between regular elements (Fig. 1c). These interface solid elements control the interaction between adjacent regular elements of the mesh. Different strategies can be adopted in relation to where and when to include the interface elements. For instance, interface elements can be introduced in the whole mesh (i.e., at the contact between all the bulk elements of the model) at the beginning of the analysis. To reduce the number of interface elements, they could be included in some regions of the mesh or materials only, where the formation of cracks is anticipated. This strategy will lead to a reduction of the computational effort, but it is necessary to have reliable beforehand information about the zone(s) and/or material(s) where cracks may be formed. It can also be possible to start the analysis with a standard mesh (i.e. with bulk element only) and, as the problem evolves, enhance the mesh with interface elements in those zones where the stress field indicates that cracks are prone to form. For this last option, techniques similar to the ones typically used in re-meshing strategies for the densification of meshes in critical zones can be adopted [11, 15, 35].

This paper focuses on the proposal of an orthotropic mechanical constitutive model for describing the behavior of the HAR elements between distinct materials with orthotropic interface characteristics. Isotropic mechanical models for HAR can be found elsewhere [22, 40].

Sánchez et al. [40] discussed in detail the main advantages of the MFT with respect to other methods based on the inclusion of special elements between standard FE, such as cohesive zone models and zero-thickness interface elements [5, 27, 32]. One key characteristic of the MFT is that the numerical analysis is entirely conducted in a continuum approach, with no need to define discrete mechanical models, or distinct integration rules and interpolation functions (as it is necessary for zero-thickness elements). Manzoli et al. [21, 22] demonstrated that the implementation of HAR elements in conjunction with proper strain-softening constitutive models allows simulating the kinematics associated with the formation of displacement discontinuities according to the continuous strong discontinuous approach [8, 29, 36, 41]. These elements can also be used to model discontinuities related to the degradation of the union between distinct materials.

As for the interface elements, we adopted two nonlinear constitutive models based on the damage theory. To represent the shear interfaces necessary to simulate the potential slip of the soil with respect to the boundary (e.g., soil-plate interphase in a drying test in the laboratory), we adopted a model defined in terms of the cohesion bond strength. To describe the interface between soil elements related to crack opening (i.e., soil-soil interface), we selected a damage model which damage criterion and softening laws are defined in terms of both, soil tensile strength and soil fracture energy. Both models are introduced in detail in the next Sects. 3.2 and 3.3. As for the bulk elements, any appropriate constitutive model can be adopted when using this technique. For the sake of simplicity, we have chosen an elastic model for the analyses presented hereafter. However, more advanced models can be adopted, if deemed convenient. No coupled diffusion processes are included in the modeling, and we simulated the shrinkage process by imposing a deformation field in the soil (bulk) elements. We considered compressive stresses and strains as positive.

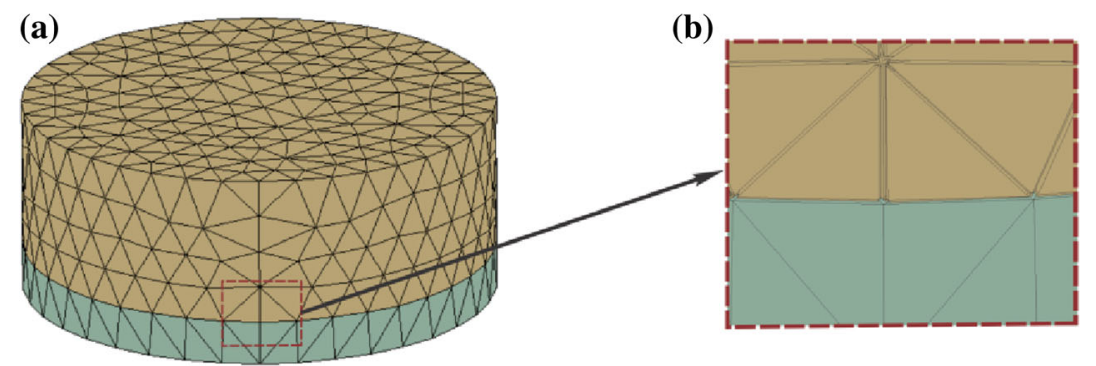

soil-soil interface elements tensile damage model

(c)

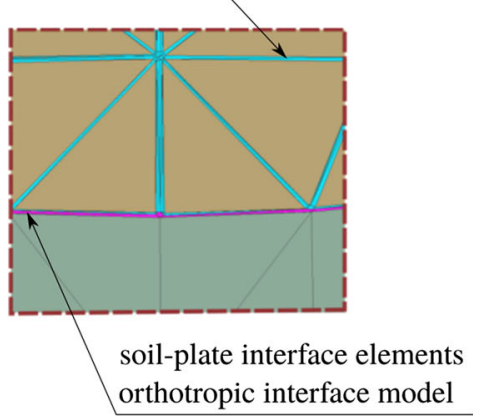

Fig. 1 Mesh fragmentation technique: a original FE mesh composed by standard (bulk) elements only, b separation of the bulk elements of the mesh, creating small gaps between them (note that only a region of the mesh is fragmented), $\mathbf{c}$ introduction of the finite elements with high aspect ratio (i.e., interface elements) in the gaps 
Sánchez et al. [40] showed the capability of the MFT to simulate the formation and propagation of desiccation cracks observed in drying soils in the laboratory by using samples of different shapes (i.e., circular, slab and rectangular plates) and also in the field. The proposed technique was able to explain and reproduce satisfactorily the effect of several factors affecting the drying behavior of soils, including (among others) influence of the specimen thickness on crack pattern, effect of sample size on crack morphology and relevance of the contact between the soil and plate on crack spacing and orientation. The strong influence of the contact surface between materials on the final crack pattern was observed in both experimental tests available in the literature (e.g., [34]) and numerical modeling [40]. This particular aspect is analyzed in detail in Sect. 4.3 using both isotropic and anisotropic mechanical models for representing the soil-plate contact surface.

\section{Interface mechanical models}

Two nonlinear orthotropic constitutive mechanical models for the interface elements based on the damage theory are presented in this section. Section 3.1 discusses general aspects and presents the basic equations related to the interface solid elements typically used in the MFT. A Mohr-Coulomb-type orthotropic damage model is proposed in Sect. 3.2 to represent the orthotropic shear interfaces necessary to simulate the potential slip between materials (e.g., soil-plate interfaces in drying tests involving orthotropic characteristics). A damage model (similar to the one described in [40]) is introduced in Sect. 3.3 to describe the interfaces between soil elements related to crack opening (i.e., soil-soil interface). An elastic model was adopted for the bulk elements; more advanced constitutive laws can be incorporated if necessary. Finally, Sect. 3.4 describes (very briefly) the algorithm used for implementing the suggested interface mechanical models into the FE computer code.

\subsection{Interface solid finite element}

Figure 2 shows the 4-node tetrahedron FE adopted as an example hereafter. The total strain ( $(\varepsilon)$ can be split into:

$\boldsymbol{\varepsilon}=\boldsymbol{\varepsilon}+\underbrace{\frac{1}{h}(\mathbf{n} \otimes \llbracket \mathbf{u} \rrbracket)^{s}}_{\hat{\varepsilon}}$

where the tensor $\hat{\boldsymbol{\varepsilon}}$ is associated with the strain components that depend on the element height $(h)$, while $\tilde{\boldsymbol{\varepsilon}}$ contains the rest of the components. The symmetric part of $(\cdot)$ is

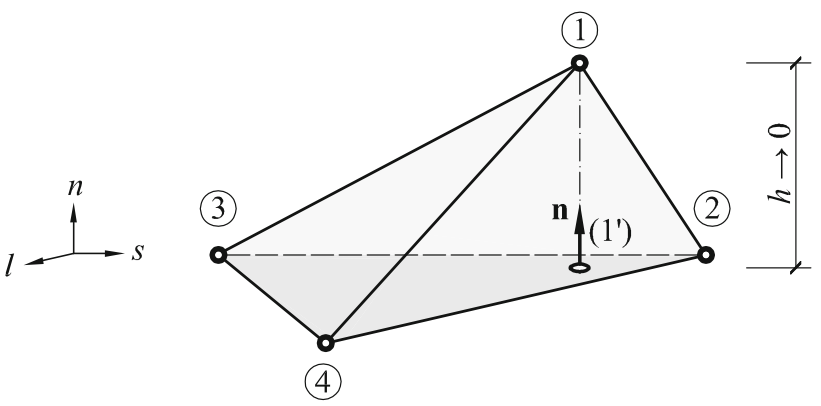

Fig. 2 Tetrahedron finite element with high aspect ratio

represented by $(\cdot)^{s} ; \mathbf{n}$ is the unit vector normal to the element base; $\otimes$ denotes a dyadic product; and $\llbracket \mathbf{u} \rrbracket$ is the relative displacement vector between node 1 and $1^{\prime}$ (i.e., its projection on the element base, Fig. 2). As shown in Manzoli et al. [21], when $h$ tends to zero the tensor $\hat{\boldsymbol{\varepsilon}}$ is related almost exclusively to the relative displacement between node 1 and $1^{\prime}$, and the relative displacement $\llbracket \mathbf{u} \rrbracket$ is a measure of the displacement discontinuity (strong discontinuity).

Considering that the local axis $n$ is perpendicular to the element base (Fig. 2), the unbounded part of the strain tensor can be written as

$\hat{\boldsymbol{\varepsilon}}=\left[\begin{array}{ccc}\hat{\varepsilon}_{n n} & \hat{\varepsilon}_{n s} & \hat{\varepsilon}_{n l} \\ \hat{\varepsilon}_{n s} & \hat{\varepsilon}_{S s} & \hat{\varepsilon}_{l l} \\ \hat{\varepsilon}_{n l} & \hat{\varepsilon}_{s l} & \hat{\varepsilon}_{l l}\end{array}\right]=\frac{1}{h}\left[\begin{array}{ccc}\llbracket u \rrbracket_{n} & \frac{1}{2} \llbracket u \rrbracket_{s} & \frac{1}{2} \llbracket u \rrbracket_{l} \\ \frac{1}{2} \llbracket u \rrbracket_{s} & 0 & 0 \\ \frac{1}{2} \llbracket u \rrbracket_{l} & 0 & 0\end{array}\right]$

where $\llbracket u \rrbracket_{n}, \llbracket u \rrbracket_{s}$ and $\llbracket u \rrbracket_{l}$ are the components of the displacement jump according to the local $(n, s, l)$ coordinate system. More details can be found elsewhere [21-23].

\subsection{Orthotropic interface model}

To reproduce the orthotropic character of interface surfaces, a model that considers two damage criteria is proposed hereafter in terms of the shear components of the stress tensor parallel to the base of the interface element, as follows:

$\phi_{s}=\left\|\sigma_{n s}\right\|+\alpha_{s} \sigma_{n n}-q_{s}\left(r_{s}\right) \leq 0$

$\phi_{l}=\left\|\sigma_{n l}\right\|+\alpha_{l} \sigma_{n n}-q_{l}\left(r_{l}\right) \leq 0$

where $q_{s}$ and $q_{l}$ are stress-like internal variables, $r_{s}$ and $r_{l}$ are strain-like internal variables, and $\alpha_{s}$ and $\alpha_{l}$ are the model parameters that control the influence of the component of the normal stress tensor $\sigma_{n n} \leq 0$.

The model is expressed by the following constitutive equation relating the components of the stress tensor $(\boldsymbol{\sigma})$ and the strain tensor $(\boldsymbol{\varepsilon})$ according to the local coordinate system $(n, s, l)$ : 


$$
\begin{gathered}
\underbrace{\left[\begin{array}{ccc}
\sigma_{n n} & \sigma_{n s} & \sigma_{n l} \\
\sigma_{n s} & \sigma_{s s} & \sigma_{s l} \\
\sigma_{n l} & \sigma_{s l} & \sigma_{l l}
\end{array}\right]}_{\boldsymbol{\sigma}}=\underbrace{\left[\begin{array}{ccc}
\bar{\sigma}_{n n} & 0 & 0 \\
0 & \bar{\sigma}_{s s} & \bar{\sigma}_{s l} \\
0 & \bar{\sigma}_{s l} & \bar{\sigma}_{l l}
\end{array}\right]}_{\overline{\boldsymbol{\sigma}}_{n s l}} \\
+\left(1-d_{s}\right) \underbrace{\left[\begin{array}{ccc}
0 & \bar{\sigma}_{n s} & 0 \\
\bar{\sigma}_{n s} & 0 & 0 \\
0 & 0 & 0
\end{array}\right]}_{\bar{\tau}_{s}}+\left(1-d_{l l}\right) \underbrace{\left[\begin{array}{ccc}
0 & 0 & \bar{\sigma}_{n l} \\
0 & 0 & 0 \\
\bar{\sigma}_{n l} & 0 & 0
\end{array}\right]}_{\bar{\tau}_{l}}
\end{gathered}
$$

where $d_{s} \in[0,1]$ and $d_{l} \in[0,1]$ are the scalar damage variables,

$\left[\begin{array}{lll}\bar{\sigma}_{n n} & \bar{\sigma}_{n s} & \bar{\sigma}_{n l} \\ \bar{\sigma}_{n s} & \bar{\sigma}_{s s} & \bar{\sigma}_{s l} \\ \bar{\sigma}_{n l} & \bar{\sigma}_{s l} & \bar{\sigma}_{l l}\end{array}\right]=\mathbb{C}:\left[\begin{array}{lll}\varepsilon_{n n} & \varepsilon_{n s} & \varepsilon_{n l} \\ \varepsilon_{n s} & \varepsilon_{s s} & \varepsilon_{s l} \\ \varepsilon_{n l} & \varepsilon_{s l} & \varepsilon_{l l}\end{array}\right]$

where $\overline{\boldsymbol{\sigma}}$ is the elastic stress tensor (i.e., the stresses related to the intact, undamaged, cross section, also known as effective stress in damage models) and $\mathbb{C}$ is the isotropic fourth-order elastic tensor.

Dividing Eqs. (3) and (4) by $\left(1-d_{s}\right)$ and $\left(1-d_{l}\right)$, respectively, the damage criteria can be expressed in terms of the elastic stresses, as:

$\bar{\phi}_{s}=\left\|\bar{\sigma}_{n s}\right\|+\alpha_{s} \bar{\sigma}_{n n}-r_{s} \leq 0$

$\bar{\phi}_{l}=\left\|\bar{\sigma}_{n l}\right\|+\alpha_{l} \bar{\sigma}_{n n}-r_{l} \leq 0$

where $r_{s}=\left(q_{s}-d_{s} \alpha_{s} \bar{\sigma}_{n n}\right) /\left(1-d_{s}\right)$ and $r_{l}=\left(q_{l}-d_{l} \alpha_{l} \bar{\sigma}_{n n}\right)$ $/\left(1-d_{l}\right) . \bar{\sigma}_{n s}$ and $\bar{\sigma}_{n l}$ are the shear components of the elastic stress tensor, which is evaluated from the strains by linear elastic relationship (6). They are related to the stress components $\sigma_{n s}$ and $\sigma_{n l}$ by means of constitutive Eq. (5), so that $\sigma_{n s}=\left(1-d_{s}\right) * \bar{\sigma}_{n s}$ and $\sigma_{n l}=\left(1-d_{l}\right) * \bar{\sigma}_{n l}$. The corresponding strain-like internal variables, $r_{s}$ and $r_{l}$, are obtained (after some algebra) from the following explicit evolution laws:

$$
\begin{aligned}
& r_{s}=\max _{\tau \in[0, t]}\left[\left\|\bar{\sigma}_{n s}(\tau)\right\|+\alpha_{s} \bar{\sigma}_{n n}(\tau), q_{s 0}\right] \\
& r_{l}=\max _{\tau \in[0, t]}\left[\left\|\bar{\sigma}_{n l}(\tau)\right\|+\alpha_{l} \bar{\sigma}_{n n}(\tau), q_{l 0}\right]
\end{aligned}
$$

where $t$ is the pseudo-time associated with the loading process. According to Eqs. (9) and (10), the variables $r_{s}$ and $r_{l}$ will adopt the maximum value associated with the corresponding elastic stresses (i.e., $\left\|\bar{\sigma}_{n s}\right\|+\alpha_{s} \bar{\sigma}_{n n}$, and $\left\|\bar{\sigma}_{n l}\right\|+\alpha_{l} \bar{\sigma}_{n n}$, respectively) to be reached during the loading process, starting from the initial values (i.e., $q_{\text {so }}$ and $q_{t o}$, respectively), which are regarded as material properties. Note that these variables are explicitly evaluated, since they depend on the components of the elastic components only, which are obtained explicitly from the strain components, via linear elastic relationship (6). Once these internal variables are obtained for each time step during the loading process, the damage variables can be evaluated from the following damage evolution rules in terms of the internal variables $r_{s}$ and $r_{l}$ :

$d_{s}\left(r_{s}\right)=\frac{r_{s}-q_{s}}{r_{s}-\alpha_{s} \bar{\sigma}_{n n}}$

$d_{l}\left(r_{l}\right)=\frac{r_{l}-q_{l}}{r_{l}-\alpha_{l} \bar{\sigma}_{n n}}$.

Then, the stresses can be calculated by Eq. (5).

The Kuhn-Tucker relations can be expressed as:

$\bar{\phi}_{s, l}<0, \quad \dot{r}_{s, l}>0, \quad \dot{r}_{s, l} \bar{\phi}_{s, l}=0$.

The constitutive model is completed with the consistency condition, as follows: (a)

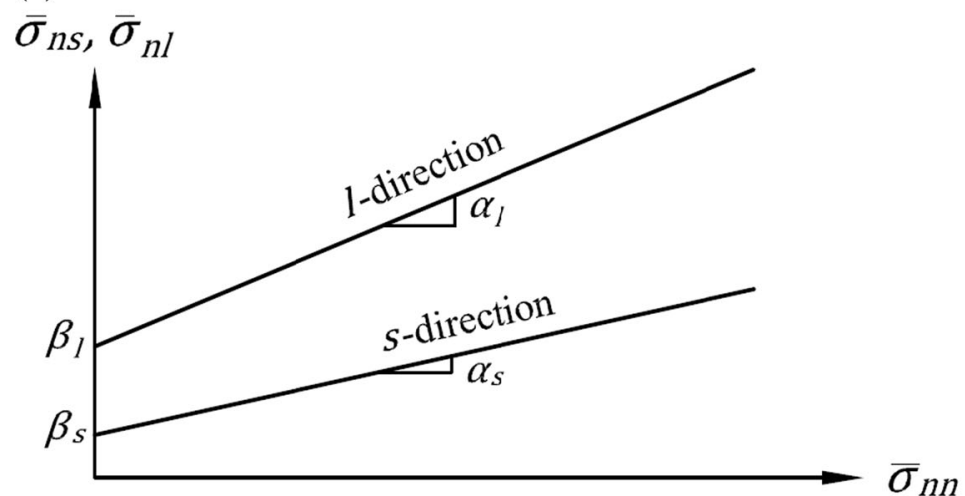

(b)

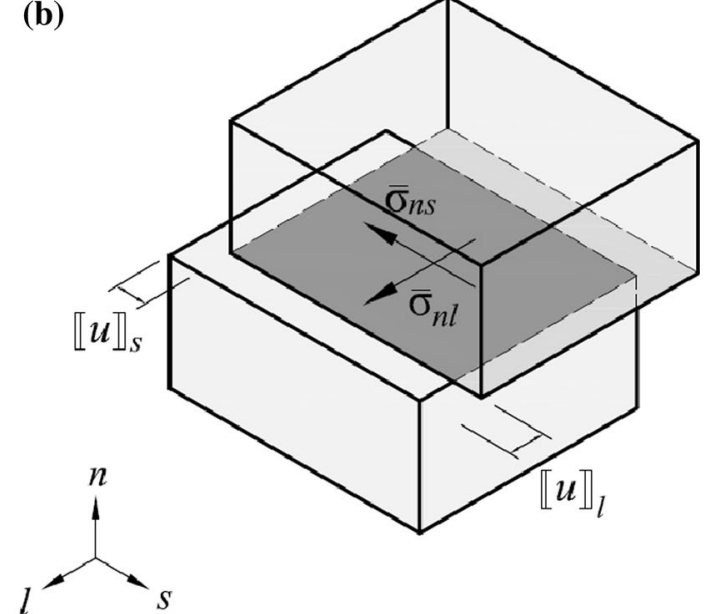

Fig. 3 a Schematic representation of the orthotropic model with the option to define different strengths in two orthogonal directions, b plot showing the sliding at the interface between materials, with the corresponding displacement jumps in two orthogonal directions 
$\dot{r}_{s, l} \dot{\bar{\phi}}_{s, l}=0 \quad$ if $\bar{\phi}_{s, l}=0$.

To represent the soil-plate interface, the stress-like variables are assumed to be constant:

$q_{s}\left(r_{s}\right)=q_{s 0}=\beta_{s}$

$q_{l}\left(r_{l}\right)=q_{l 0}=\beta_{l}$

where $\beta_{s}$ and $\beta_{l}$ are the cohesive bonding strengths of the soil-plate interface in directions $s$ and $l$, respectively. Figure $3 \mathrm{a}$ presents a schematic representation of this model.

\subsection{Tension damage model}

The tension damage model used to describe cracks formation in the soil has the same structure as the previous model and other ones published before (e.g., [10, 33]). The constitutive equation in this case is given by:

$\boldsymbol{\sigma}=(1-d) \overline{\boldsymbol{\sigma}}$

and the damage criterion is written in terms of the component of the stress normal to the element base:

$\phi=\sigma_{n n}-q(r) \leq 0$

or in terms of the elastic stress as:

$\bar{\phi}=\bar{\sigma}_{n n}-r \leq 0$.

The evolution of the stress-like variable is given by an exponential softening law of the form:

$q(r)=f_{\mathrm{t}} e^{\frac{f_{\mathrm{t}}^{2}}{G_{\mathrm{f}}} h\left(1-\frac{r}{f_{\mathrm{t}}}\right)}$

where $E$ is the Young's modulus, $f_{\mathrm{t}}$ is the tensile strength, and $G_{\mathrm{f}}$ is the mode I fracture energy of the soil. More details can be found elsewhere (i.e., [22, 40]).

\subsection{Implicit-explicit integration scheme}

The implicit-explicit (IMPL-EX) integration algorithm proposed by Oliver et al. [30] was adopted in this work to update the stresses using the new orthotropic law. The IMPL-EX is a robust, efficient and stable numerical scheme to integrate mechanical models. A similar approach to the one suggested in Manzoli et al. [22] was followed in this paper. Table 1 summarizes the main stages associated with the update of the stresses at a pseudo-time step $t_{(n+1)}$ using the orthotropic damage models with the IMPL-EX method. Note that any other algorithm (i.e., implicit or explicit) could be adopted for the integration of the proposed constitutive model. However, based on our experience, the IMPL-EX method is very appropriate to deal with this type of problem.

\section{Application cases}

This section is related to the application of the proposed model to different cases involving orthotropic interfaces. The first case consists of a series of 'synthetic benchmarks' based on theoretical cases aimed at checking the capability of the proposed model to reproduce different possible orientations of the orthotropic interface with respect to the loading direction. The second application focuses on the validation of the orthotropic mechanical model by using laboratory tests involving the shearing of soil specimens against plates especially grooved in different directions. Finally, the suggested model is applied to solve the slab test reported in Peron et al. [34].

All the examples were modeled using four-node tetrahedral finite elements. The Mohr-Coulomb-type orthotropic damage model was used to describe the behavior of the interfaces between different materials. Following the MFT, interface elements with high aspect ratio were introduced between regular elements, which were modeled assuming a linear elastic behavior.

\subsection{Synthetic benchmarks}

The theoretical cases are based on two square blocks with an interface between them (Fig. 4). One block (i.e., block A) is fixed to a wall with displacements restricted in all directions. In the other block (i.e., block B) a displacement field is imposed simultaneously on the four 'free nodes' in the vertical plane with an inclination of $45^{\circ}$, as shown in Fig. 5a. It is assumed that the interface between blocks is grooved in one direction, restricting the relative movement between blocks in the orthogonal direction to the grooves. Four different cases are study to explore the capability of the proposed technique to reproduce the orthotropic behavior of the interfaces. The different cases are generated by placing the grooves in different orientations with respect to the direction of the imposed displacement field. The orthotropic model can simulate this type of problem by assigning different strengths related to the two dominant directions existing in this problem. In all the cases considered below it is assumed that the strength in the direction perpendicular to the grooves is much higher than the one related to the direction of the grooves. Table 2 lists the material properties of the blocks and interface. A total of 16 nodes and 30 elements were used to simulate this problem, 24 of them are 3D bulk elements (i.e., to model the 2 blocks), and 6 are HAR interface elements. They were placed at the interface between the 2 blocks only to simulate the contact behavior.

In the first case the grooves coincide with the direction of the imposed displacement (Fig. 5b). Under this 
Table 1 IMPL-EX integration scheme for the orthotropic damage model

INPUT: $\boldsymbol{\varepsilon}_{(n+1)}, \bar{\sigma}_{n n_{(n)}}, r_{s_{(n)}}, \Delta r_{s_{(n)}}, r_{l_{(n)}}, \Delta r_{l_{(n)}}$

(i) Transform the current strain tensor obtaining its components according to the local coordinate system $(n, s, l)$

(ii) Compute the elastic stress tensor

$$
\boldsymbol{\sigma}_{(n+1)}=\mathbb{C}: \boldsymbol{\varepsilon}_{(n+1)}
$$

(iii) Check the loading-unloading conditions

(a) in direction $S$

$$
\operatorname{IF}\left(\left\|\bar{\sigma}_{n s_{(n+1)}}\right\|+\alpha_{s} \bar{\sigma}_{n n_{(n+1)}}-r_{s_{(n)}} \leq 0\right), \text { THEN }
$$

Keep the threshold: $r_{s_{(n+1)}}:=r_{s_{(n)}}$

ELSE

Update the damage threshold: $r_{s_{(n+1)}}:=\left\|\bar{\sigma}_{n s_{(n+1)}}\right\|+\alpha_{s} \bar{\sigma}_{n n_{(n+1)}}$

END IF

(b) in direction $l$

$\mathrm{IF}\left(\left\|\bar{\sigma}_{n l_{(n+1)}}\right\|+\alpha_{l} \bar{\sigma}_{n n_{(n+1)}}-r_{l_{(n)}} \leq 0\right)$, THEN

Keep the threshold: $r_{l_{(n+1)}}:=r_{l_{(n)}}$

ELSE

Update the damage threshold: $r_{l_{(n+1)}}:=\left\|\bar{\sigma}_{n l_{(n+1)}}\right\|+\alpha_{l} \bar{\sigma}_{n n_{(n+1)}}$

END IF

(iv) Compute the strain-like internal variable increments

$$
\Delta r_{s_{(n+1)}}:=r_{s_{(n+1)}}-r_{s_{(n)}} ; \Delta r_{l_{(n+1)}}:=r_{l_{(n+1)}}-r_{l_{(n)}}
$$

(v) Compute the explicit linear extrapolation of the strain-like internal variable

$$
\tilde{r}_{s_{(n+1)}}:=r_{s_{(n)}}+\frac{\Delta t_{(n+1)}}{\Delta t_{(n)}} \Delta r_{s_{(n)}} ; \quad \tilde{r}_{l_{(n+1)}}:=r_{l_{(n)}}+\frac{\Delta t_{(n+1)}}{\Delta t_{(n)}} \Delta r_{l_{(n)}}
$$

(vi) Update the damage variables

$$
\tilde{d}_{s_{(n+1)}}:=\frac{\tilde{r}_{s_{(n+1)}}-\beta_{s}}{\tilde{r}_{s_{(n+1)}}-\alpha_{s} \bar{\sigma}_{n n_{(n)}}} ; \quad \tilde{d}_{l_{(n+1)}}:=\frac{\tilde{r}_{l_{(n+1)}}-\beta_{l}}{\tilde{r}_{l_{(n+1)}}-\alpha_{l} \bar{\sigma}_{n n_{(n)}}}
$$

(vii) Compute the stress tensor according to Eq. (5)

$$
\boldsymbol{\sigma}_{(n+1)}:=\overline{\boldsymbol{\sigma}}_{n s l_{(n+1)}}+\left(1-\tilde{d}_{s_{(n+1)}}\right) \overline{\boldsymbol{\tau}}_{s_{(n+1)}}+\left(1-\tilde{d}_{l_{(n+1)}}\right) \overline{\boldsymbol{\tau}}_{l_{(n+1)}}
$$

(viii) Rotate the stress tensor obtaining its components according to the global coordinate system $(x, y, z)$

OUTPUT: $\boldsymbol{\sigma}_{(n+1)}, \bar{\sigma}_{n n_{(n+1)}}, r_{s_{(n+1)}}, \Delta r_{s_{(n+1)}}, r_{l_{(n+1)}}, \Delta r_{l_{(n+1)}}$ 


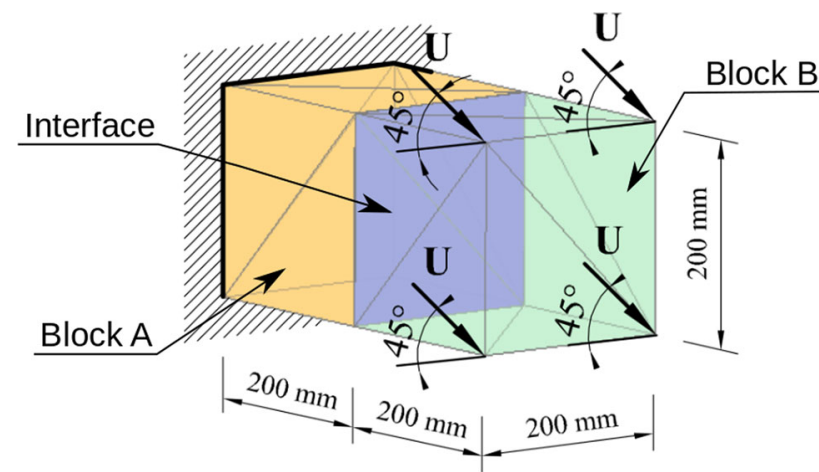

Fig. 4 Geometry and boundary conditions related to the proposed synthetic benchmark

condition, the relative movement between blocks is not restricted, this implies that block A does not deform, while block B freely slides along the grooves at $45^{\circ}$ (i.e., without deforming). In the second case the grooves are placed perpendicular to the direction of the imposed displacement field (Fig. 5c). This implies that the relative movement between the two blocks is fully restricted and the two blocks deform as a monolithic body. The third case corresponds to the grooves oriented in the vertical direction (i.e., at $45^{\circ}$ with respect to the imposed displacement field). In this case both blocks deform as a single piece in the $x$ direction, while sliding occurs in the $y$ direction (Fig. 5d). In the last case the grooves are oriented in the $x$ direction (Fig. 5e), therefore the blocks deform as a single body in the $y$ direction, and the adopted interface enables the sliding in the $x$ direction.

The top drawings in Fig. 5b-e show the orientation of the grooves adopted in the different cases. The bottom ones show the deformed blocks after applying the displacement field (i.e., solid line), alongside their initial positions before (a)

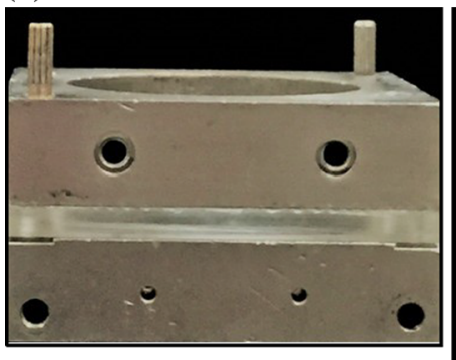

(b)

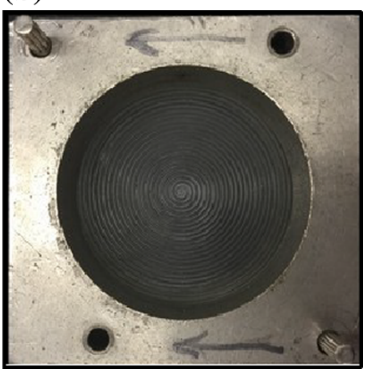

Fig. 6 Modified direct shear box for testing soil-plate interface strength: a front photograph, b top photograph

loading. It can be observed that the orthotropic model manages to reproduce very satisfactory the anticipated behavior under these different conditions, allowing sliding between blocks in the direction along the grooves only.

Once the capability of the orthotropic model to qualitatively reproduce the behavior of orthotropic interfaces through these challenging synthetic benchmarks was checked, the ability of the model to simulate the experimental behavior of this type of problem was investigated, as explained in the next section.

\subsection{Experimental validation of the orthotropic model}

A conventional direct shear apparatus was adapted to study the shear strength between soil-plate interfaces. The modification basically consisted of placing a square plate between the top and the bottom portions of the direct shear box device. Figure 6a shows a front view of the modified apparatus with the acrylic plate placed in between the two portions of the shear box. Figure $6 \mathrm{~b}$ corresponds to a top

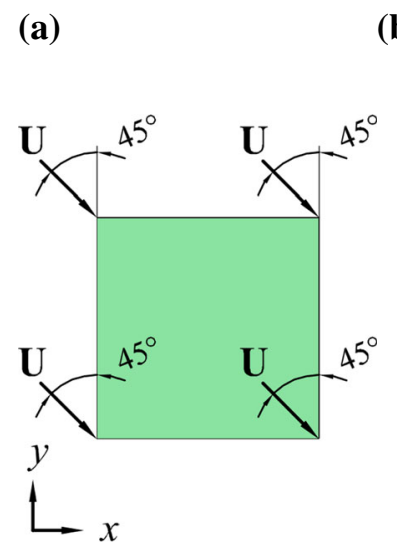

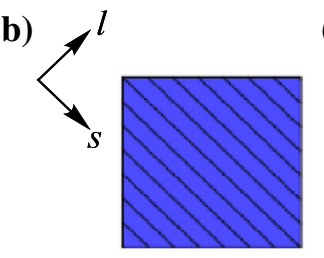

(c)
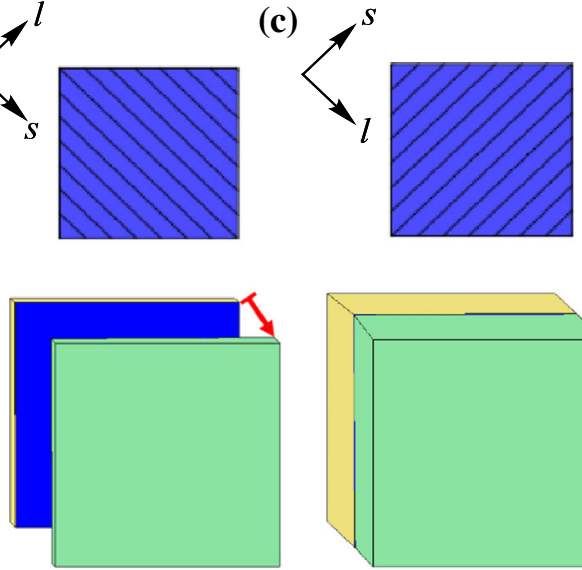

(d)
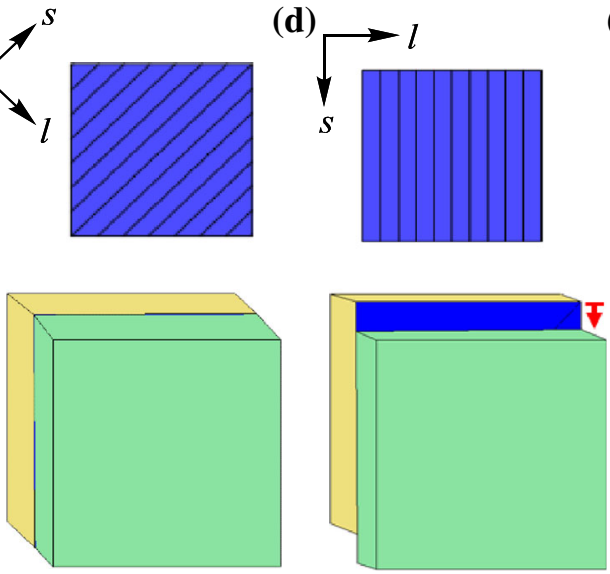

(e)
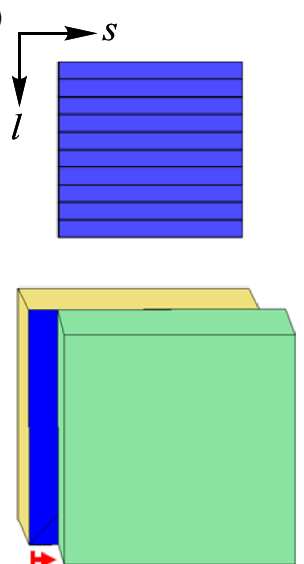

Fig. 5 a Frontal view of the synthetic benchmark, b grooves parallel to the imposed displacement field, $\mathbf{c}$ grooves perpendicular to the imposed displacements, $\mathbf{d}$ grooves in the $y$ direction and $\mathbf{e}$ grooves in the $x$ direction. In figures $\mathbf{b}-\mathbf{e}$ the top drawing shows the orientation of the grooves for the different studied cases, and the bottom ones show the deformed blocks (indicating with arrows the direction of the block movement) 
Table 2 Properties of the blocks and interface used to study the synthetic benchmarks

\begin{tabular}{llllllll}
\hline Element & $E(\mathrm{GPa})$ & $v$ & $\alpha_{s}$ & $\alpha_{l}$ & $\beta_{s}(\mathrm{MPa})$ & $\beta_{l}$ (MPa) & Constitutive model \\
\hline Blocks (bulk) & 30 & 0.0 & - & - & - & - & Elastic \\
Block-block interfaces & 30 & 0.0 & 0.00 & 0.00 & 90 & 300 & Orthotropic damage \\
\hline
\end{tabular}

view of the adapted box. The study of soil interfaces of unsaturated soils was conducted before by Miller and Hamid [24] and Hamid and Miller [13] using a similar approach.

Acrylic square bases ( $88.9 \mathrm{~mm}$ side) and $6 \mathrm{~mm}$ high were grooved to study the effect of different interface textures on the shear strength. Triangular grooves $1 \mathrm{~mm}$ deep and spaced every $1.5 \mathrm{~mm}$ were machined following two main patterns: (1) spiral and (2) straight. Three sets of direct shear tests were conducted with these two plates: (1) using the circular indentations (Fig. 7a); (2) orienting the straight grooves perpendicular to the shearing direction (Fig. 7b); and (3) placing the straight grooves parallel to the shearing direction (Fig. 7c). The orientation of the local coordinate system $(s, l)$ depends on the direction of the grooves, where ' $s$ ' coincides with the direction of the (a)
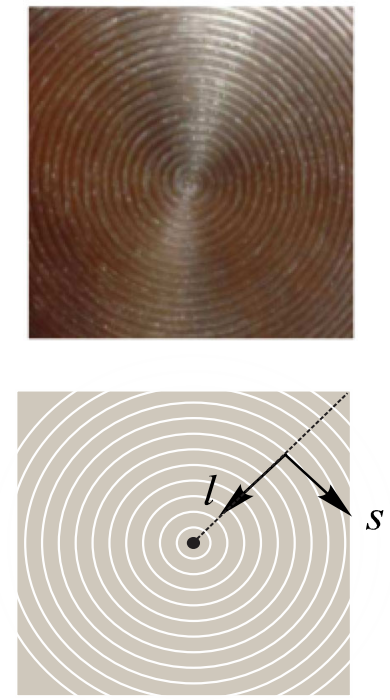

(b)
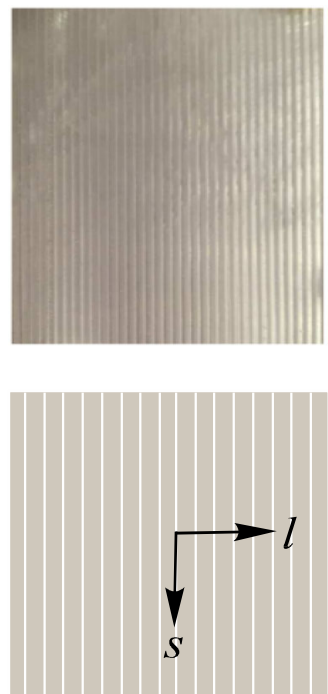

(c)
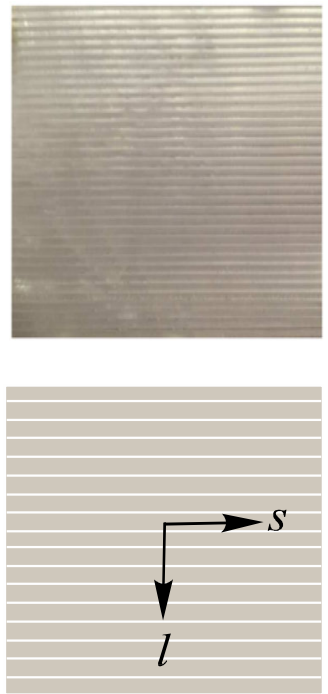

(d)
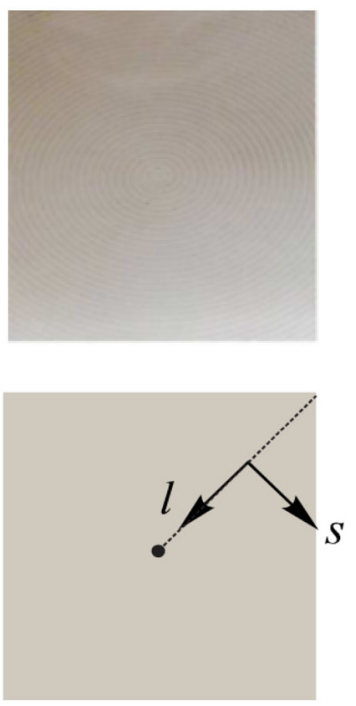

Fig. 7 Different types of plate surfaces: a spiral grooves, $\mathbf{b}$ grooves oriented orthogonal with respect to the shear direction, $\mathbf{c}$ grooves oriented parallel to the shear direction and $\mathbf{d}$ smooth surface

(a)

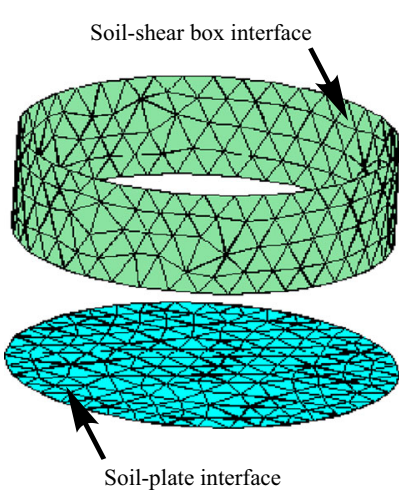

(b)

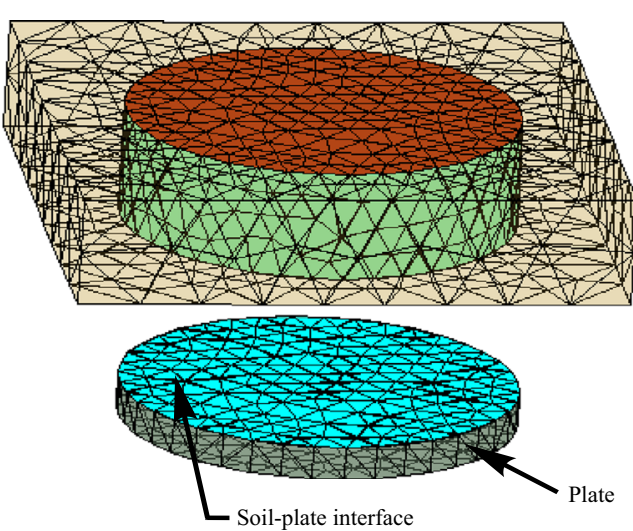

(c)

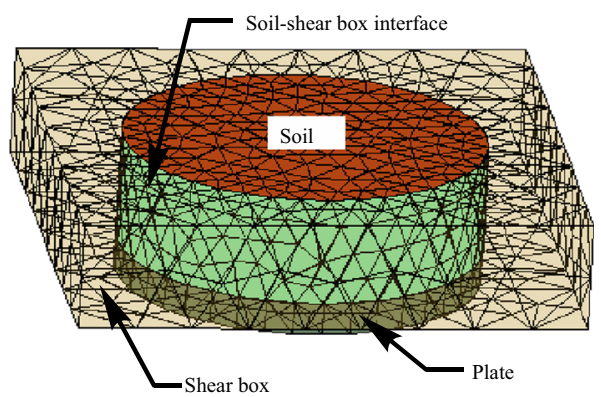

Fig. 8 Modeling of direct shear tests, main components: a interfaces formed with HAR FEs, b interfaces, shear box, plates and soil and $\mathbf{c}$ final FE mesh with all the components to simulate the test 
(a)
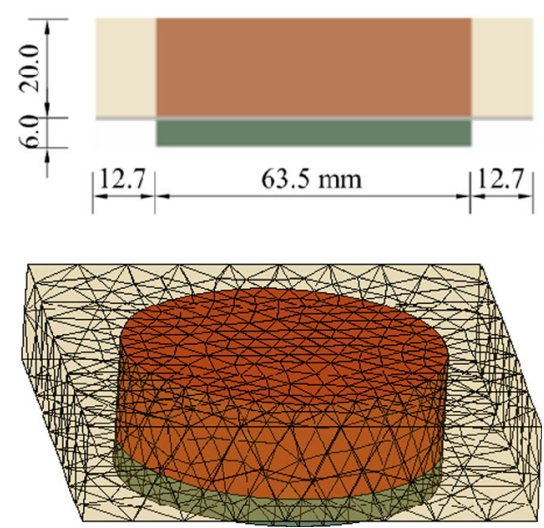

(b)
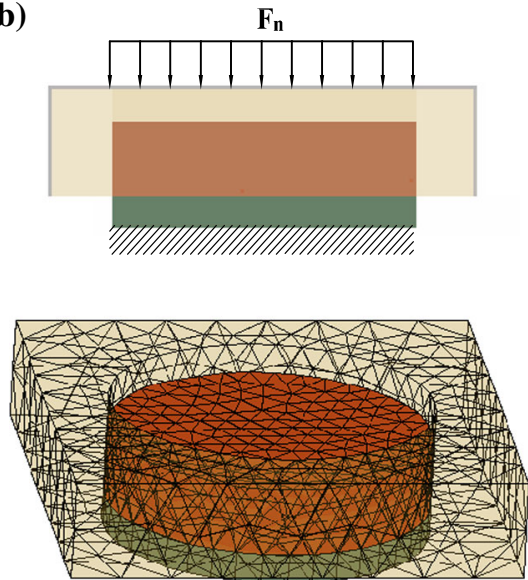

(c)

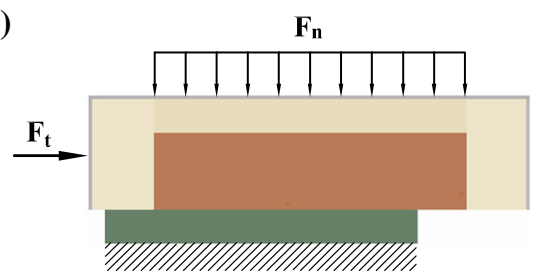

Fig. 9 Modeling of the direct shear test of soil-plate interfaces: a before testing, $\mathbf{b}$ deformed specimen after applying the vertical load $F_{n}$ at the top and $\mathbf{c}$ deformed specimen after shearing
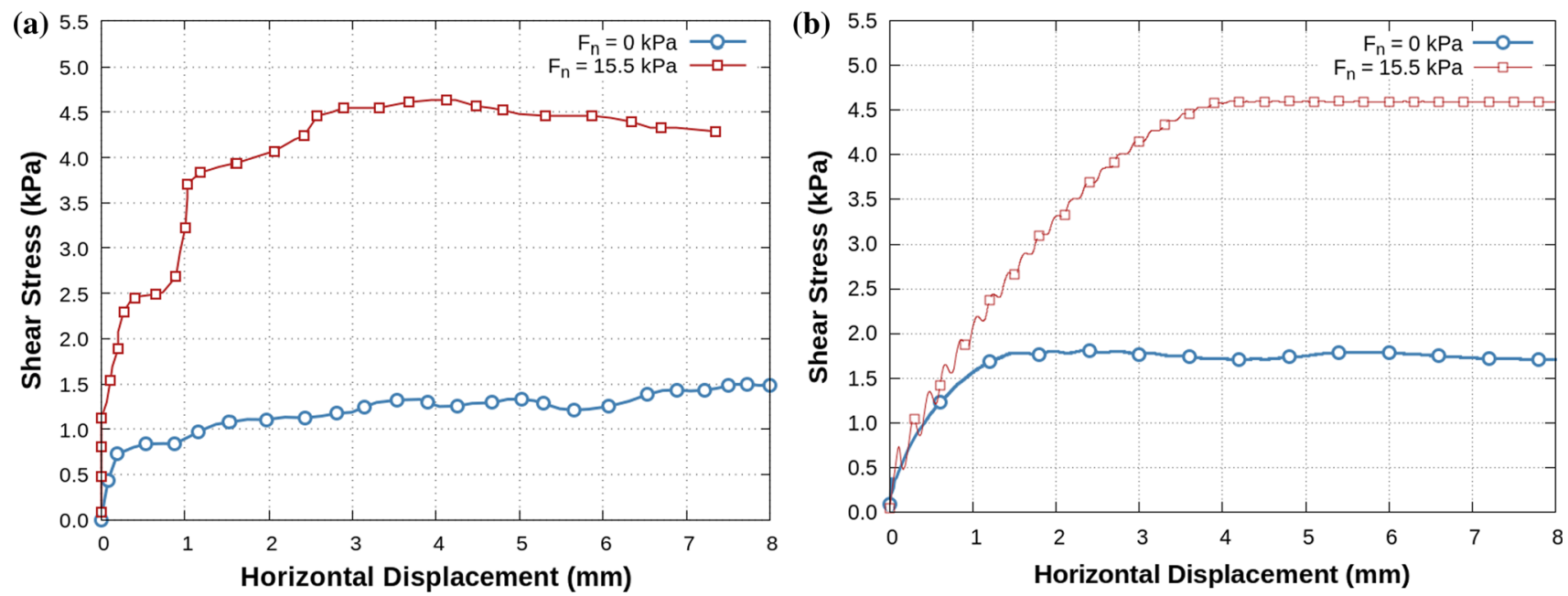

Fig. 10 Shear force against horizontal displacements when load is applied perpendicular to the grooves (i.e., Fig. 7b): a experimental results and b numerical outputs
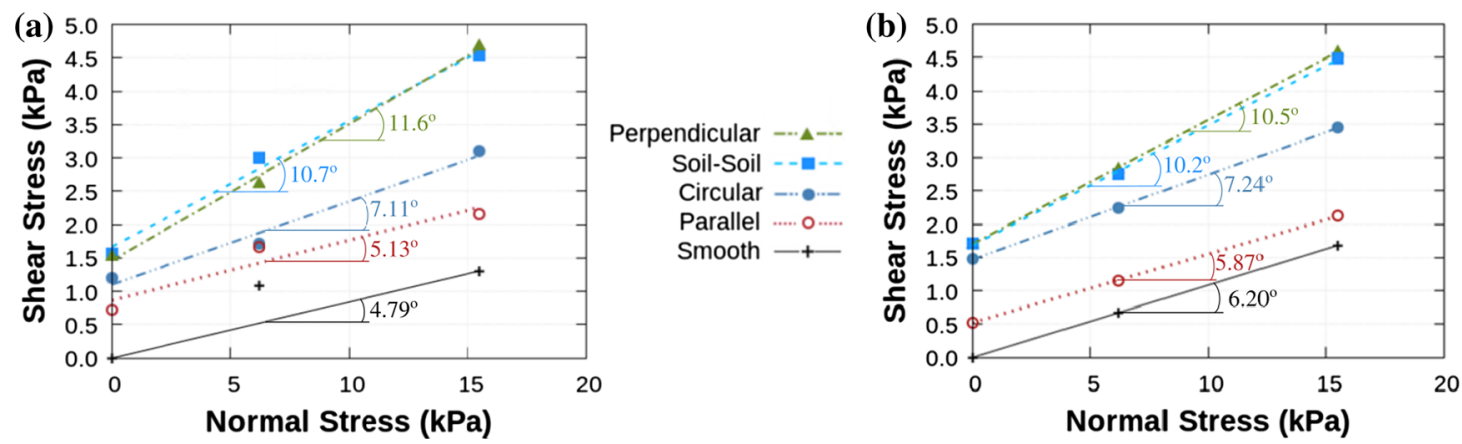

Perpendicular $-\cdots-\cdots$ Soil-Soil --m--

Circular -...--

Parallel $\ldots . . .0 \ldots . . .$.

Smooth -+

Fig. 11 Failure envelopes related to the direct shear tests: a experimental and $\mathbf{b}$ numerical results

grooves and ' $l$ ' is perpendicular to them. Note that for the circular case, the coordinate system depends on the point under consideration and therefore ' $l$ ' corresponds to the radial direction and ' $s$ ' to the tangential one (Fig. 7a). Two additional sets of direct shear tests were conducted, one of them using a smooth plate (Fig. 7d) and another one to study the soil-soil-shear strength, without interface (i.e., a standard direct shear test). 
Table 3 Material properties of soil, plate, shear box and interfaces used in the numerical analysis of the interface shear test

\begin{tabular}{llllllll}
\hline Element & $E(\mathrm{kPa})$ & $v$ & $\alpha_{s}$ & $\alpha_{l}$ & $\beta_{s}(\mathrm{kPa})$ & $\beta_{l}$ (kPa) & Constitutive model \\
\hline Soil (bulk) & 40 & 0.2 & - & - & - & - & Elastic \\
Plate & $210 \times 10^{6}$ & 0.2 & - & - & - & - & Elastic \\
Shear box & $210 \times 10^{6}$ & 0.2 & - & - & - & - & Elastic \\
Soil-shear box interfaces & 40 & 0.0 & 0.00 & 0.00 & 0.00 & 0.00 & Orthotropic damage \\
Soil-plate interfaces (soil-soil) & 40 & 0.0 & 0.19 & 0.19 & 1.67 & 1.67 & Orthotropic damage \\
Soil-plate interfaces (smooth) & 40 & 0.0 & 0.11 & 0.11 & 0.00 & 0.00 & Orthotropic damage \\
Soil-plate interfaces (perpendicular) & 40 & 0.0 & 0.11 & 0.19 & 0.50 & 1.67 & Orthotropic damage \\
Soil-plate interfaces (parallel) & 40 & 0.0 & 0.11 & 0.19 & 0.50 & 1.67 & Orthotropic damage \\
Soil-plate interfaces (circular) & 40 & 0.0 & 0.11 & 0.19 & 0.50 & 1.67 & Orthotropic damage \\
\hline
\end{tabular}

(a)

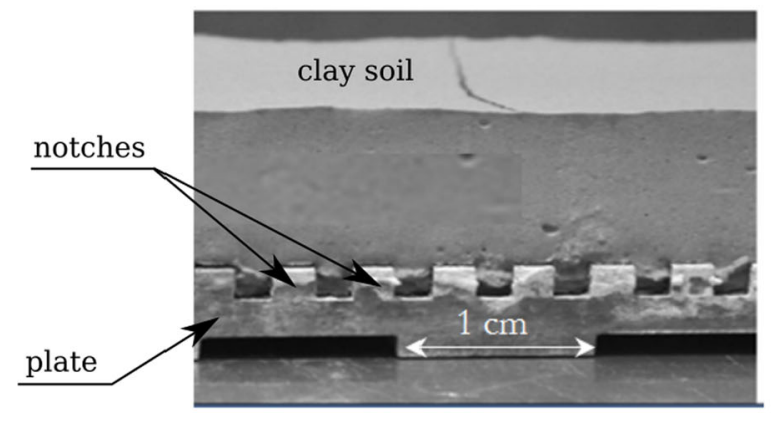

(b) clay soil

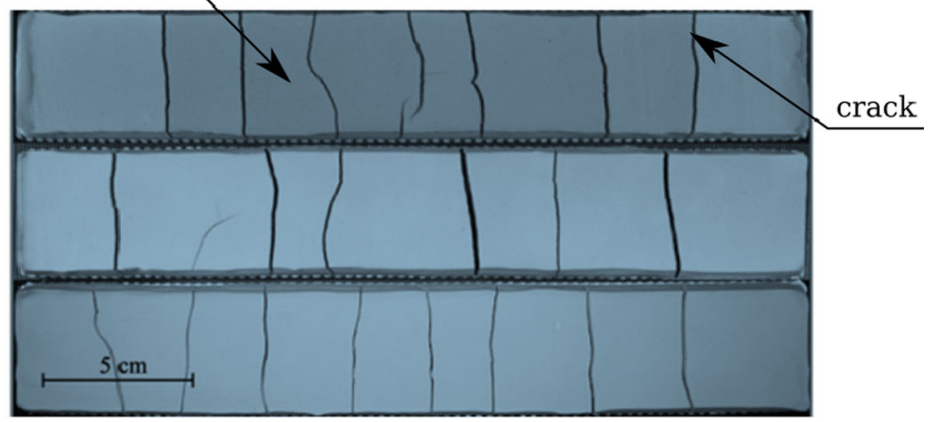

Fig. 12 Drying tests on saturated clay samples prepared in slab plates (Peron et al. [32]): a detail of the soil slap contact showing the 2-mm notches, b typical examples of crack formation in 3 different soil samples (ㄷ 2009 Canadian Science Publishing or its licensors. Reproduced with permission)

The test protocol was the usual one for the direct shear test [4]. The shear tests were conducted for the four sets of experiments (i.e., circular indentations, straight grooves parallel and orthogonal to the shearing direction, smooth plates and soil-soil strength) at three different normal stresses: (1) $0 \mathrm{kPa}$, (2) $6.2 \mathrm{kPa}$ and (3) $15.5 \mathrm{kPa}$. The specimens were sheared at a very low rate (i.e., $0.018 \mathrm{~mm} /$ $\min )$ to emulate drained conditions. Shearing was continued until the peak value of the shear force was clearly obtained or until it becomes almost constant. The tests were conducted up to a maximum displacement of around $9 \mathrm{~mm}$.

The tests were simulated using the MFT incorporating the orthotropic mechanical model to account for the different textures of the soil-plate interfaces discussed above. Figure 8 presents the main components incorporated in the modeling of these tests, i.e., interfaces (Fig. 8a), shear box, plates and soil (Fig. 8b). Figure 8c shows all the model components together. The total number of nodes is 3928 , with a total number of elements of 12,154, where 8542 corresponds to bulk elements and 3612 to interface elements, which were placed at the plate-soil and (shear) box-soil contacts only. A mesh convergence study was conducted to investigate the effect of the mesh size on the model outputs. It was observed that the adopted mesh yield results are (almost) identical to the ones obtained with finer ones. Figure $9 \mathrm{a}$ shows the geometry of the problem and the adopted mesh before loading. Figure $9 \mathrm{~b}$ presents the deformed mesh after applying the vertical load $\left(F_{n}\right)$, and Fig. $9 \mathrm{c}$ shows the mesh after shearing the sample. Figure 10a presents the evolution of shear stress against the horizontal displacements for two experiments carried out under two different loading conditions, as follows: under a vertical stress of $15.5 \mathrm{kPa}$ and without a vertical load. In both cases the grooves were placed orthogonal to the shear direction. In both tests the shear stress increased (almost) monotonically until reaching the maximum value and then remained (approximately) constant as the specimen continued deforming. As expected, the maximum shear depends on the applied vertical stress. Similar patterns of behavior were observed in the other experiments performed in the context of this study.

Figure 11a presents a summary in terms of failure envelopes of all the shear tests conducted under the 


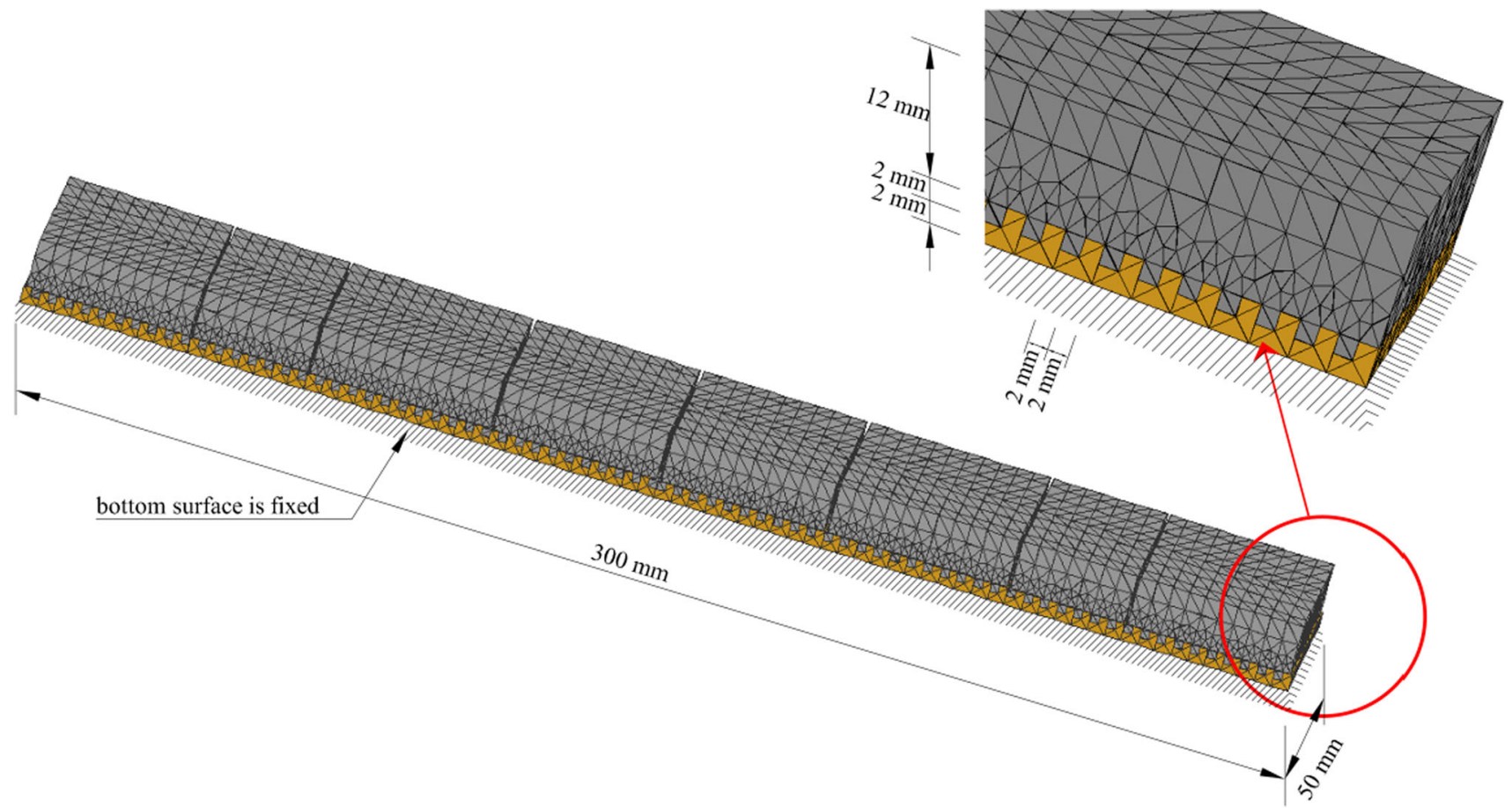

Fig. 13 3D modeling of the drying tests on saturated clay samples prepared in slab plates discretizing the indentation at the soil-plate interface

different conditions discussed above. As it could be anticipated, the higher strength is obtained for the soil-soil experiments and the lower one for those tests involving the smooth plate. The soil-plate strength for the base with the grooves oriented perpendicular to the direction of shearing is very high, similar to the soil-soil case, indicating that (under these conditions) the soil is strongly bonded to the plate and the shearing takes place between soil and soil particles mainly. As it could be anticipated, the strength for the case of indentations oriented parallel to the shear direction is lower than the orthogonal one discussed above. As expected, the results involving the circular plate are in between the ones obtained for the parallel and orthogonal cases. It is also observed that the different interfaces affect mainly the interception (i.e., $\beta$ ) of the linear envelope adopted to define shear strength, and the impact on the envelope inclination (i.e., $\alpha$ ) is relatively minor.

The following strategy was adopted to model the experiments involving grooves: two of them were used to obtain the model parameters, and the third one was reserved for the model validation. The available experimental data (i.e., Figure 11a) were used to determine the strength parameters (i.e., $\beta$ and $\alpha$ ) for the case in which the shearing direction is perpendicular and parallel to the grooves. Afterward, the circular case was used to validate the model using the same parameters adopted for the plates with straight grooves. As for the soil-soil and smooth cases, the parameters were adjusted based on the test results presented in Fig. 11a. The material properties of the soil, plate, shear box and interfaces are listed in Table 3.

Figure $11 \mathrm{~b}$ presents the results of the numerical simulations using the MFT together with the orthotropic mechanical model. It can be observed that the proposed orthotropic model is able to reproduce very satisfactorily the global experimental trends. In particular, the model manages to simulate that the strength of the case involving the circular grooves (acting as a benchmark case in this modeling) is in between the strength obtained for the grooves parallel and orthogonal to the shearing directions. (a)

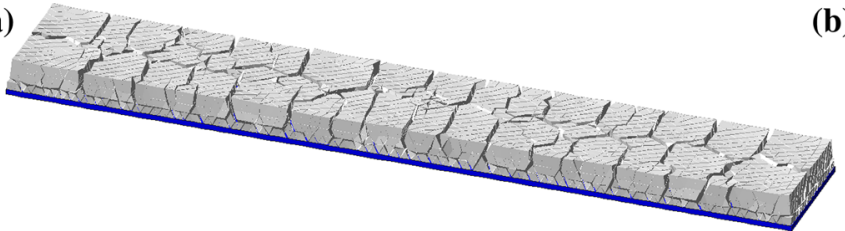

(b)

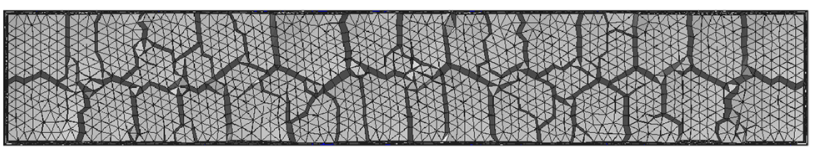

Fig. 14 Modeling the slab specimen using an isotropic interface model for the soil-plate contact surface, a 3D and b top views (respectively) 


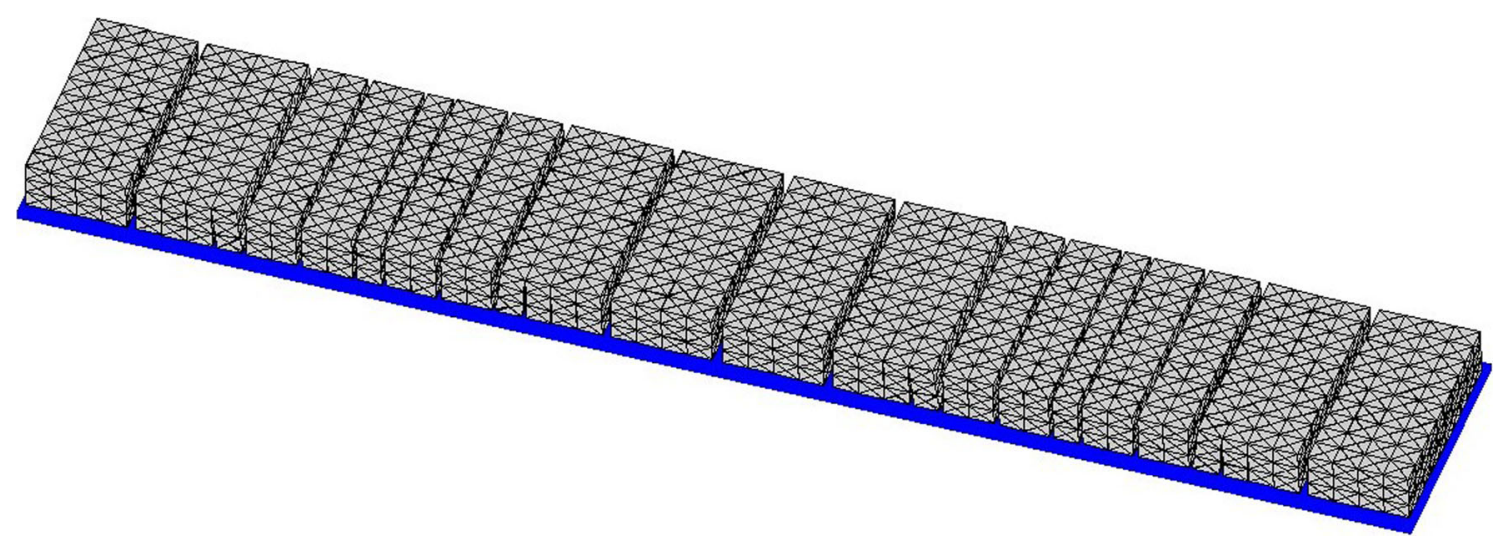

Fig. 15 Modeling the slab specimen using an orthotropic interface model for the soil-plate contact surface (structured mesh)

\subsection{Modeling a slab desiccation tests with orthotropic characteristics}

The desiccation tests conducted by Peron et al. [34] were selected to show the capabilities of the proposed approach to simulate the formation of cracks when orthotropic conditions at the soil-plate contact prevail. The experiments were based on slab samples prepared from clay slurries. Two types of contact surface were investigated:

1. smooth contact between soil and plate;

2. longitudinal restricted displacement induced by the inclusion of notches at soil-plate the contact surface (Fig. 12a).

As for the free shrinkage case (i.e., type i above), a base made of Teflon was used in combination with the application of a hydrophobic substance to prevent any displacement constrains at the soil-plate contact. Peron et al. [34] reported that all the specimens subjected to this drying condition did not crack. Sánchez et al. [40] modeled this experimental behavior using the MFT in conjunction with an isotropic constitutive model for the soil-plate interface that allows free displacements. As expected, tensile horizontal stresses in the soil did not develop and drying cracks did not form. Similar behavior (i.e., shrinkage without cracking) has been observed in other investigations involving smooth contact soil-plate surface (e.g., [44]).

In relation to the tests conducted under constrained desiccation conditions (i.e., case ii above), the slurry samples were prepared in a similar manner as in the free drying tests, but the Teflon base was replaced by a metal surface with 2-mm spaced parallel notches (Fig. 12a). These notches created a constraint at the bottom surface, in the longitudinal direction only. Samples subjected to drying under these conditions experienced one-directional cracks only and in the direction parallel to the constraint (Fig. 12b). In the following sections this test is modeled using two different approaches: (1) first the simulation of the experiment is conducted including the actual geometry of the notches in the mesh discretization, together with the adoption of an isotropic constitutive model for the soilplate interface; (2) then, a planar plate surface is assumed in conjunction with the proposed orthotropic mechanical model to account for the orthotropic characteristic of this problem.

In both cases the drying process is simulated by imposing an increasing uniform volumetric strain field to the bulk elements of the soil, which is equivalent to the shrinkage induced during water evaporation in the soil mass. There is not an external load in addition to the selfweight. It is further assumed that the plate is fixed to the

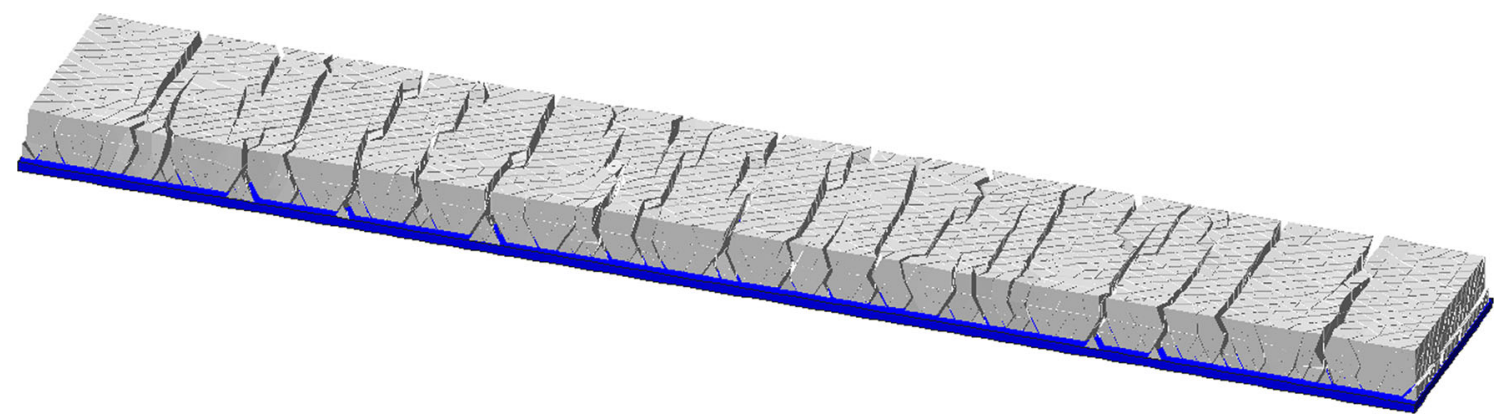

Fig. 16 Modeling the slab specimen using an orthotropic interface model for the soil-plate contact surface (unstructured mesh) 
Table 4 Material properties of soil and plate used in the simulations of desiccation cracks

\begin{tabular}{llllllllll}
\hline Element & $E(\mathrm{MPa})$ & $v$ & $\alpha_{s}$ & $\alpha_{l}$ & $\beta_{s}(\mathrm{kPa})$ & $\beta_{l}(\mathrm{kPa})$ & $f_{\mathrm{t}}(\mathrm{kPa})$ & $G_{\mathrm{f}}(\mathrm{N} / \mathrm{m})$ & Constitutive model \\
\hline Soil (bulk) & 4 & 0.2 & - & - & - & - & - & - & Elastic \\
Plate & $210 \times 10^{3}$ & 0.2 & - & - & - & - & - & - & Elastic \\
Soil-soil interfaces & 4 & 0.0 & - & - & - & - & 4.0 & 0.4 & Tensile damage \\
Soil-plate interfaces & 4 & 0.0 & 0.00 & 0.00 & 3.20 & 3.2 & - & - & Isotropic damage \\
Soil-plate interfaces & 4 & 0.0 & 0.00 & 0.00 & 0.96 & 3.2 & - & - & Orthotropic damage \\
\hline
\end{tabular}

ground and therefore cannot move in any direction during the drying process, as indicated in Fig. 13.

\subsubsection{Modeling the contact between materials with an isotropic mechanical model}

The MFT technique was successfully applied to model this test by exactly replicating the geometry of the base used in the experiments including the 2-mm notches, as indicated in the zoom of Fig. 13. No noticeable moisture gradients were measured in those experiments [34]. Therefore, an uniform deformation field was imposed to simulate the shrinkage process. As in the experiments, the model reproduces one-directional cracks only, which are parallel to the direction of the constraint with no longitudinal cracks (Fig. 13).

To successfully model these experiments, it was necessary to explicitly include the indentations in the simulations. There are two main drawbacks associated with this type of solution: (1) it is very laborious and time-consuming to discretize the exact geometry of the interface (i.e., 2-mm indentation in this case); and (2) a quite dense mesh is required to capture all the details associated with this particular soil-plate contact. Denser meshes are related to an increase in the degrees of freedom of the problem, resulting in more expensive numerical analyses in terms of CPU time. Note that the simulation of this problem without including the notches and using an isotropic mechanical model for the interface (as the one described in [40]) leads to unrealistic results with the formation of transversal and longitudinal cracks (Fig. 14), which do not correspond to the observed behavior in this experiment or in similar ones.

\subsubsection{Modeling a slab desiccation test using the orthotropic interface model}

The last application case is related to the modeling of the drying of the slab specimen discussed before, but now using the orthotropic mechanical model to implicitly consider the displacement restriction imposed by the notches present at the soil-plate interface. Therefore, a planar surface at this contact surface was assumed and the parameters of the orthotropic mechanical model are such that the longitudinal relative displacements are restricted and the soil can slide (almost freely) in the transversal direction only. This arrangement induces the formation of transversal cracks only as shown in Fig. 15, and as it was observed in the experiments.

After a quick inspection of Figs. 13 and 15, it can be concluded that the results obtained with the mesh that includes a detailed geometrical representation of the orthotropic soil-plate contact surface (i.e., Fig. 13) are (almost) identical to the ones obtained with a model that combines a (simple) planar plate surface and the orthotropic mechanical law proposed in this work (i.e., Fig. 15). The implicit discretization of the orthotropic characteristics of a contact surfaces between different materials through a mechanical model that includes the dependence of the materials strength in terms of the direction of the analysis has two main advantages: (1) requires a simpler mesh (reducing therefore the time associated with its preparation) and (2) yields a mesh with less number of nodes with the associated reduction in the degree of freedom of the problem (saving therefore CPU time).

If we now compare the results obtained with these two different approaches (i.e., Figs. 13, 15) against the experimental observations (i.e., Fig. 12b), it is evident that the perfectly straight vertical cracks simulated by these two models are not totally realistic. This outcome is related to the adoption of structured meshes to model this problem, which tend to induce the propagation of cracks through the well-defined, regular and straight (vertical) contacts between elements. A more realistic simulation of drying cracks can be achieved by using an unstructured mesh to model this problem. Figure 16 presents the $3 \mathrm{D}$ view of a model based on an unstructured mesh of 73,048 nodes and 128,853 elements, where 24,741 corresponds to bulk elements and 104,112 to interface elements (which were placed at the plate-soil contact and between standard bulk soil elements, i.e., all the domain but the plate). Table 4 lists the model parameters adopted in the simulation. This model output resembles quite accurately the actual cracked 
soil. As shown in Sánchez et al. [40], no noticeable dependence of the crack pattern on the adopted mesh size is observed when using the MFT. The difference in the aspect of the crack networks observed between the perfectly regular one, shown in Fig. 15 (i.e., obtained with a structured mesh), and the apparently more realistic crack network presented in Fig. 16 (i.e., obtained with an unstructured mesh) is because cracks tend to be aligned with the main orientations generally present in structured meshes, while in the case of an unstructured meshes there is not a predominant direction for the crack to propagate.

\section{Conclusions}

The difference in directional strengths at the contact between materials generally arrives from the presence of textures at the interfaces. The incorporation of these interface characteristics between materials is instrumental for a realistic solution of several engineering problems. However, the explicit discretization of these textures in the modeling is generally time-consuming (as sometimes relatively small structural details need to be represented), and also the associated meshes are very dense (with the corresponding demand in CPU time).

In this work, an orthotropic mechanical model for soils developed in the framework of the mesh fragmentation technique was proposed. The full mathematical framework alongside the suggested numerical algorithm for its numerical implementation in a FE code was discussed in detail. The capability of the model to reproduce different plausible loading conditions involving orthotropic interfaces was checked by means of tailored synthetic benchmarks. A very good performance of the model was observed in the four cases analyzed. This paper also deals with the experimental validation of the suggested model. Direct shear tests involving soil and plates with different textures were conducted to study the dependence of the interface strength properties on orthotropic characteristics. The comparisons between the proposed model and experimental results were very satisfactory, proving the ability of the model to capture the observed response of orthotropic interfaces involving shearing directions perpendicular and parallel to straight grooves, as well as soil shearing with respect to a plate grooved following a circular pattern and also a smooth plate. Finally, the model was applied to analyze a boundary value problem related to the drying behavior of a soil subjected to shrinkage with restriction of displacement in one direction. Also in this case the model was able to reproduce very well the developments of drying cracks observed in the experiment.
Acknowledgements Marcelo Sánchez would like to acknowledge the financial support from the Sao Paulo Research Foundation (FAPESP, proc. 2016/19479-2). The authors also acknowledge the support from the National Council for Scientific and Technological Development (CNPq, proc. 234003/2014-6).

\section{References}

1. Abu Al-Rub RK (2007) Prediction of micro and nanoindentation size effect from conical or pyramidal indentation. Mech Mater 39:787-802. https://doi.org/10.1016/j.mechmat.2007.02.001

2. Amarasiri AL, Kodikara JK, Costa S (2011) Numerical modelling of desiccation cracking. Int $\mathrm{J}$ Numer Anal Methods Geomech 35:82-96. https://doi.org/10.1002/nag.894

3. Asahina D, Houseworth JE, Birkholzer JT et al (2014) Hydromechanical model for wetting/drying and fracture development in geomaterials. Comput Geosci 65:13-23. https://doi.org/10.1016/j. cageo.2013.12.009

4. ASTM D3080 (2011) American Society for Testing and Materials. Stand Test Method Direct Shear Test Soils Under Consol Drained Cond. https://doi.org/10.1520/E0606-04E01

5. Caballero A, Carol I, López CM (2006) A meso-level approach to the 3D numerical analysis of cracking and fracture of concrete materials. Fract Eng Mater Struct 29:979-991

6. Caggiano A, Etse G, Martinelli E (2012) Zero-thickness interface model formulation for failure behavior of fiber-reinforced cementitious composites. Comput Struct 98-99:23-32. https:// doi.org/10.1016/j.compstruc.2012.01.013

7. Carol I, Prat PC, López CM (1997) Normal/shear cracking model: application to discrete crack analysis. J Eng Mech 123:765-773. https://doi.org/10.1061/(ASCE)07339399(1997)123:8(765)

8. Chen Q, Andrade JE, Samaniego E (2011) AES for multiscale localization modeling in granular media. Comput Methods Appl Mech Eng 200:2473-2482. https://doi.org/10.1016/j.cma.2011. 04.022

9. Etse G, Caggiano A, Vrech S (2012) Multiscale failure analysis of fiber reinforced concrete based on a discrete crack model. Int J Fract 178:131-146. https://doi.org/10.1007/s10704-012-9733-z

10. Ferrara A, Pandolfi A (2008) Numerical modelling of fracture in human arteries. Comput Methods Biomech Biomed Eng 11:553-567. https://doi.org/10.1080/10255840701771743

11. Geißler G, Netzker C, Kaliske M (2010) Discrete crack path prediction by an adaptive cohesive crack model. Eng Fract Mech 77:3541-3557. https://doi.org/10.1016/j.engfracmech.2010.04. 029

12. Gens A, Carol I, Alonso EE (1989) An interface element formulation for the analysis of soil-reinforcement interaction. Comput Geotech 7:133-151. https://doi.org/10.1016/0266352X(89)90011-6

13. Hamid TB, Miller GA (2009) Shear strength of unsaturated soil interfaces. Can Geotech J 46:595-606. https://doi.org/10.1139/ T09-002

14. Hauseux P, Roubin E, Seyedi DM, Colliat JB (2016) FE modelling with strong discontinuities for 3D tensile and shear fractures: application to underground excavation. Comput Methods Appl Mech Eng 309:269-287. https://doi.org/10.1016/j.cma. 2016.05.014

15. Khoei AR, Moslemi H, Majd Ardakany K et al (2009) Modeling of cohesive crack growth using an adaptive mesh refinement via the modified-SPR technique. Int J Fract 159:21-41. https://doi. org/10.1007/s10704-009-9380-1 
16. Lakshmikantha MR, Prat PC, Ledesma A (2009) Image analysis for the quantification of a developing crack network on a drying soil. Geotech Test J. https://doi.org/10.1520/GTJ102216

17. Li B, Jiang Y, Mizokami T et al (2014) Anisotropic shear behavior of closely jointed rock masses. Int J Rock Mech Min Sci 71:258-271. https://doi.org/10.1016/j.ijrmms.2014.07.013

18. Liu C, Tang CS, Shi B, Bin Suo W (2013) Automatic quantification of crack patterns by image processing. Comput Geosci 57:77-80. https://doi.org/10.1016/j.cageo.2013.04.008

19. López CM, Carol I, Aguado A (2008) Meso-structural study of concrete fracture using interface elements. I: numerical model and tensile behavior. Mater Struct 41:583-599. https://doi.org/10. 1617/s11527-007-9314-1

20. López CM, Carol I, Aguado A (2008) Meso-structural study of concrete fracture using interface elements. II: compression, biaxial Brazilian test. Mater Struct 41:601-620

21. Manzoli OL, Gamino AL, Rodrigues EA, Claro GKS (2012) Modeling of interfaces in two-dimensional problems using solid finite elements with high aspect ratio. Comput Struct 94-95:70-82. https://doi.org/10.1016/j.compstruc.2011.12.001

22. Manzoli OL, Maedo MA, Bitencourt LAG, Rodrigues EA (2016) On the use of finite elements with a high aspect ratio for modeling cracks in quasi-brittle materials. Eng Fract Mech. https://doi.org/ 10.1016/j.engfracmech.2015.12.026

23. Manzoli OL, Maedo MA, Rodrigues EA, Bittencourt TN (2014) Modeling of multiple cracks in reinforced concrete members using solid finite elements with high aspect ratio. In: Bicaninc N, Mang H, Meschke G, de Borst R (eds) Computational modelling of concrete structures (EURO-C 2014, St. Anton am Arlberg, Austria). CRC Press, Balkema

24. Miller GA, Hamid TB (2007) Direct shear apparatus for unsaturated soil interface testing. ASTM Geotech Test J 30:182-191

25. Misra A (1999) Micromechanical model for anisotropic rock joints. J Geophys Res 104:23-175. https://doi.org/10.1029/ 1999JB900210

26. Molinari JF, Gazonas G, Raghupathy R et al (2007) The cohesive element approach to dynamic fragmentation: the question of energy convergence. Int J Numer Methods Eng 69:484-503. https://doi.org/10.1002/nme.1777

27. Mota A, Knap J, Ortiz M (2008) Fracture and fragmentation of simplicial finite element meshes using graphs. Int $\mathrm{J}$ Numer Methods Eng 73:1547-1570. https://doi.org/10.1002/nme.2135

28. Nahlawi H, Kodikara JK (2006) Laboratory experiments on desiccation cracking of thin soil layers. Geotech Geol Eng 24:1641-1664. https://doi.org/10.1007/s10706-005-4894-4

29. Oliver J, Cervera M, Manzoli O (1999) Strong discontinuities and continuum plasticity models: the strong discontinuity approach. Int J Plast 15:319-351. https://doi.org/10.1016/S07496419(98)00073-4

30. Oliver J, Huespe AE, Blanco S, Linero DL (2006) Stability and robustness issues in numerical modeling of material failure with the strong discontinuity approach. Comput Methods Appl Mech Eng 195:7093-7114. https://doi.org/10.1016/j.cma.2005.04.018
31. Pan PZ, Rutqvist J, Feng XT, Yan F (2014) TOUGH-RDCA modeling of multiple fracture interactions in caprock during $\mathrm{CO}_{2}$ injection into a deep brine aquifer. Comput Geosci 65:24-36. https://doi.org/10.1016/j.cageo.2013.09.005

32. Pandolfi A, Ortiz M (2002) An efficient adaptive procedure for three-dimensional fragmentation simulations. Eng Comput 18:148-159. https://doi.org/10.1007/s003660200013

33. Pandolfi A, Weinberg K (2011) A numerical approach to the analysis of failure modes in anisotropic plates. Eng Fract Mech 78:2052-2069. https://doi.org/10.1016/j.engfracmech.2011.03. 021

34. Peron H, Hueckel T, Laloui L, Hu LB (2009) Fundamentals of desiccation cracking of fine-grained soils: experimental characterisation and mechanisms identification. Can Geotech J 46:1177-1201. https://doi.org/10.1139/T09-054

35. Phongthanapanich S, Dechaumphai P (2004) Adaptive Delaunay triangulation with object-oriented programming for crack propagation analysis. Finite Elem Anal Des 40:1753-1771. https:// doi.org/10.1016/j.finel.2004.01.002

36. Regueiro RA, Borja RI (1999) A finite element model of localized deformation in frictional materials taking a strong discontinuity approach. Finite Elem Anal Des 33:283-315. https://doi. org/10.1016/S0168-874X(99)00050-5

37. Rodrigues EA, Manzoli OL, Bitencourt LAG Jr et al (2017) An adaptive concurrent multiscale model for concrete based on coupling finite elements. Comput Methods Appl Mech Eng. https://doi.org/10.1016/j.cma.2017.08.048

38. Rodríguez R, Sánchez M, Ledesma A, Lloret A (2007) Experimental and numerical analysis of desiccation of a mining waste. Can Geotech J 44:644-658. https://doi.org/10.1139/t07-016

39. Sanchez M, Atique A, Kim S et al (2013) Exploring desiccation cracks in soils using a $2 \mathrm{D}$ profile laser device. Acta Geotech 8:583-596. https://doi.org/10.1007/s11440-013-0272-1

40. Sánchez M, Manzoli OL, Guimarães LJN (2014) Modeling 3-D desiccation soil crack networks using a mesh fragmentation technique. Comput Geotech 62:27-39. https://doi.org/10.1016/j. compgeo.2014.06.009

41. Simo JC, Oliver J, Armero F (1993) An analysis of strong discontinuities induced by strain-softening in rate-independent inelastic solids. Comput Mech 12:277-296. https://doi.org/10. 1007/BF00372173

42. Wen L, Tian R (2016) Improved XFEM: accurate and robust dynamic crack growth simulation. Comput Methods Appl Mech Eng 308:256-285. https://doi.org/10.1016/j.cma.2016.05.013

43. Xu XP, Needleman A (1994) Numerical simulations of fast crack growth in brittle solids. J Mech Phys Solids 42:1397-1434. https://doi.org/10.1016/0022-5096(94)90003-5

44. Zielinski M, Sánchez M, Romero E, Atique A (2014) Precise observation of soil surface curling. Geoderma 226-227:85-93. https://doi.org/10.1016/j.geoderma.2014.02.005 